\title{
Gastric Metastasis at the First Presentation of Breast Cancer May Mimic Primary Carcinoma: A Case Report and Literature Review
}

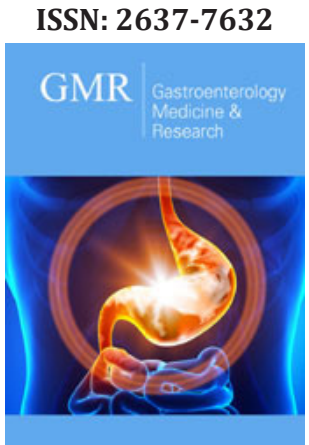

*Corresponding author: Jiayu Wang, Department of Medical Oncology, National Cancer Center/National Clinical Research Center for Cancer/ Cancer Hospital, Chinese Academy of Medical Sciences and Peking Union Medical College, China

Submission: 侮 September 24, 2020

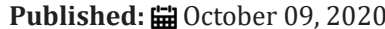

Volume 5 - Issue 2

How to cite this article: Yiqun Han, Pin Zhang, Qing Li, Fei Ma, Jiayu Wang, et al. Gastric Metastasis at the First Presentation of Breast Cancer May Mimic Primary Carcinoma: A Case Report and Literature Review. Gastro Med Res. 5(2). GMR.000609. 2020.

DOI: 10.31031/GMR.2020.05.000609

Copyright@ Jiayu Wang, This article is distributed under the terms of the Creative Commons Attribution 4.0 International License, which permits unrestricted use and redistribution provided that the original author and source are credited.
Yiqun Han, Pin Zhang, Qing Li, Fei Ma, Peng Yuan, Ying Fan, Yang Luo, Ruigang Caî, Shanshan Chen, Qiao Lil, Jiayu Wang** and Binghe Xu*

Department of Medical Oncology, National Cancer Center/National Clinical Research Center for Cancer/Cancer Hospital, Chinese Academy of Medical Sciences and Peking Union Medical College, China

\section{Abstract}

Gastric metastasis from breast cancer is a rare phenomenon in clinical practice, of which the disease features and natural course could be insidious and undistinguished from primary gastric carcinoma. We reported a 37-year-old female patient newly diagnosed as endocrine-dependent breast cancer with synchronous the diffuse thickness of stomach and extensive metastases in the peritoneum as well as bilateral ovaries. The endoscopic view demonstrated a sign of linitis plastic. Histopathological results from gastric biopsy showed molecular features in favor of breast origin, which could be the major source of antidiastole. The prognostic outcomes were heterogeneous among patients with receipt of sequential therapy, where the molecular mechanisms remained to be elucidated.

\section{Introduction}

Over the recent decades, more than $90 \%$ breast cancer at newly diagnosed is early-stage disease due to the development and generalization of screening mammography [1]. De novo stage IV breast cancer is defined as the advanced disease with synchronous involvement of distant metastasis, which accounts for mere $6 \%$ proportions in female breast cancer $[1,2]$. The common metastatic sites from primary breast cancer are bone and lung, whereas the metastasis to gastrointestinal (GI) tract is not frequent. This involvement at the first presentation of primary disease, considering the reported length timeframe to secondary lesions, could be fairly rare and sticky for antidiastole, especially in company with diffuse abdominal metastases including ovaries and peritoneum [3]. Herein, we report a case of gastric metastasis at the first presentation of breast cancer followed by a literature review of disease characteristics and natural course in clinical practice.

\section{Case report}

A 37-year-old female presented to our department with a palpable lump in the left breast which was touched at incidence in April 2019. She complaint of no symptoms other than anorexia and maransis. On physical examination, a lump in around $5 \mathrm{~cm}$ diameter, occupying the whole left breast, was detected with a crater nipple and locoregional skin ulcer, while a palpable mass was also detected in bilateral axillary and supraclavicular region. Ultrasonic examination showed a 5.9*1.8*5.3cm lump in the left breast with left axillary and supraclavicular adenopathy.

In August 2019, she underwent histopathological biopsy of puncture and the results confirmed invasive breast cancer with ipsilateral axillary nodal metastasis. The immunochemistry (IHC) of breast tissue was as follows: estrogen receptor (ER) (-), progesterone receptor (PR) $(+, 90 \%)$, HER2 $(-)$, Ki67 $(+, 10 \%)$. Then, contrast-enhanced computed tomography $(\mathrm{eCT})$ revealed that diffuse thickness of stomach fundus and corpora ventriculi (Figure 1), the mesentery, momentum majus and endometrium, in addition to the extensive metastases in bilateral ovaries, suspicious lung and pleura, bone and multiple lymph nodes. Serum biomarkers showed that CEA was 36.16 ng per milliliter (normal rage, 0.0 to 5.0), CA125 175.60U per milliliter (normal rage, 0.0 to 35.0), CA15-3 $1016.00 \mathrm{U}$ per milliliter (normal rage, 0.0 to 25.0 ), CA72-4 $40.36 \mathrm{U}$ per milliliter (normal rage, 0.0 to 6.9 ) 
and CYFRA21-1 9.85ng per milliliter (0.0-3.3). Test for hepatitis $B$, hepatitis $C$, syphilis and human immunodeficiency virus were negative. The genetic test from saliva demonstrated TP53 mutation c.1010G >A (p. Arg337His) missense mutation.
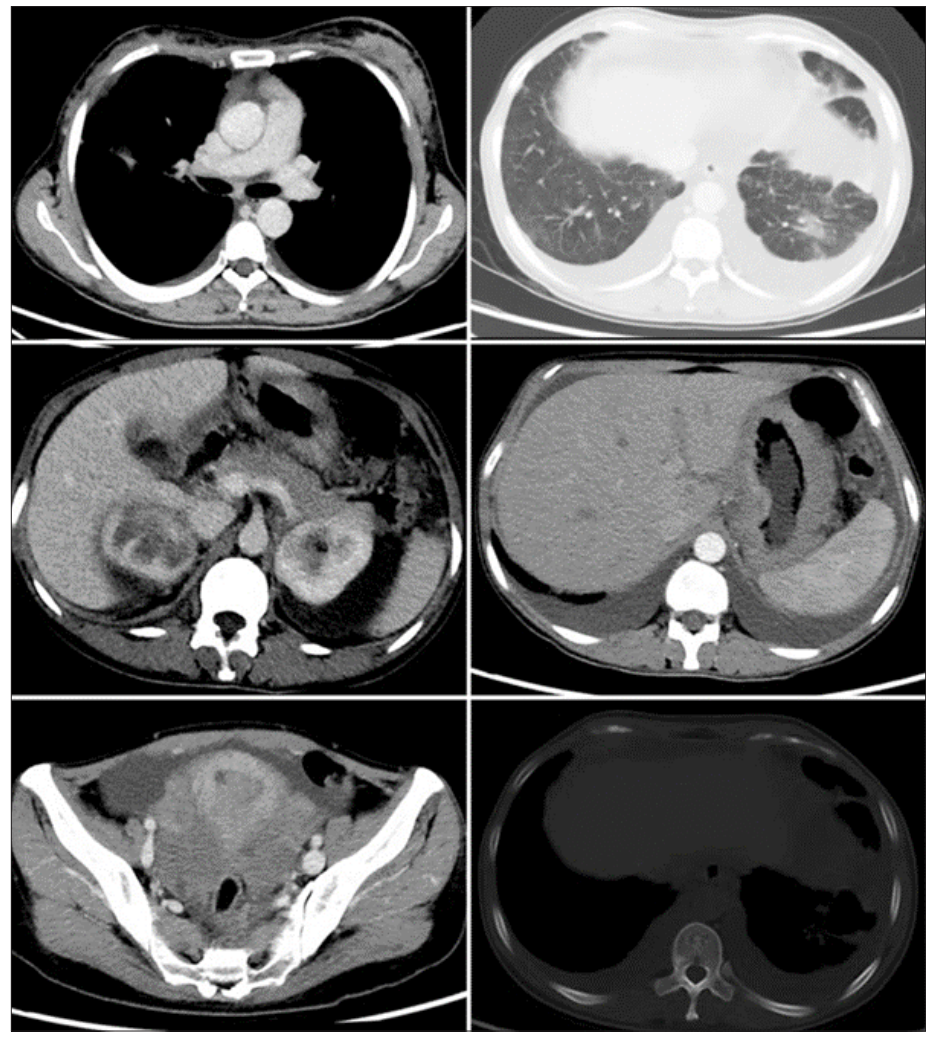

Figure 1: Computed tomography (CT) scan showing widespread metastases in stomach, ovary, diffused peritoneum and pleura, lung and bone.
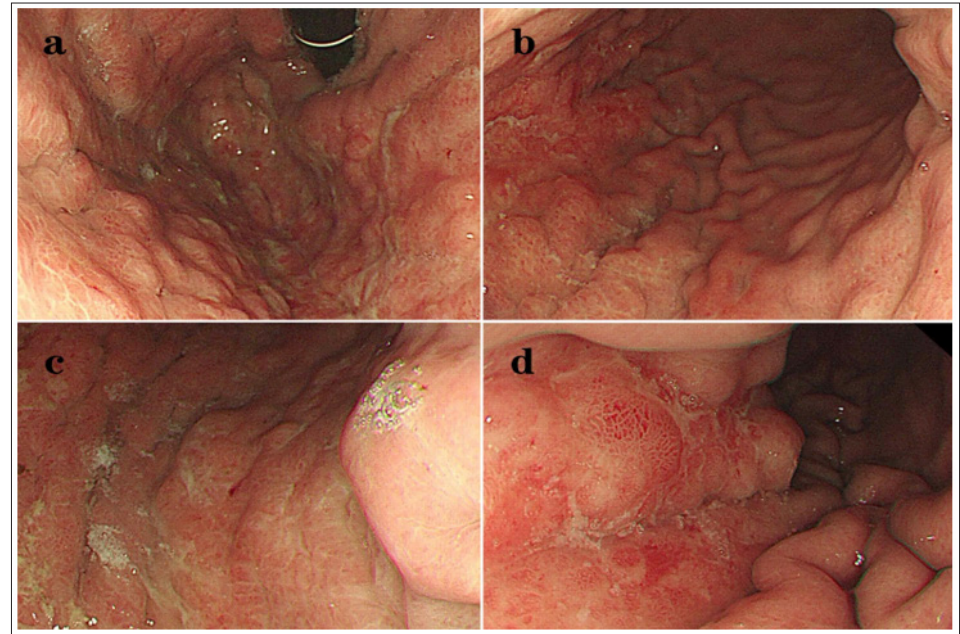

Figure 2: Endoscope showing the hypertrophic gastric folds, swelling and scattered erosion in the fundus with infiltrative appearance mimicking primary linitis plastic (a-d).

With the aim of antidiastole on gastric origin, an endoscopy examination was performed in September 2019. The endoscopic view showed a linitis plastic lesion with swelling, hyperemia, enlarged folds in the mucosa of gastric fundus and body (Figure 2). Histological results from multiple biopsies indicated a poorly differentiated carcinoma with no specific reference, while the
IHC was as follows: CK7 (3+), CDX-2 (-), CK20 (-), GATA3 (+), Mammaglobin (2+), GCDFP15 (-), ER (-), PR (+, 90\%), HER2 (2+) (Figure 3). Furthermore, the HER2 amplification was confirmed by Fluorescence in Situ Hybridization (FISH), which the ratio of HER2 to CEP17 was 6.6 and the ratio of average HER2 copies to cell was 1.9 . 


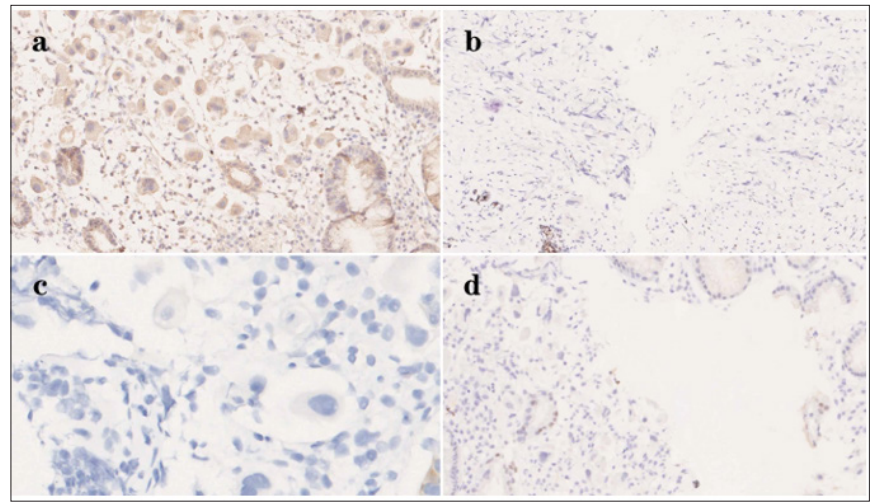

Figure 3: Immunohistochemistry results revealing gastric metastasis from breast cancer: Mammaglobin positivity (a) $(\times 20)$, GATA3 partially positivity (b) $(\times 10)$, CK20 negativity (c) $(\times 20)$ and CDX-2 negativity $(\mathrm{d})(\times 10)$.
Meanwhile, systemic therapy based on epirubicin 90mg/ $\mathrm{m}^{2}$ and paclitaxel liposomes $175 \mathrm{mg} / \mathrm{m}^{2}$ every 21 days was administrated on September 20 ${ }^{\text {th }}, 2019$. In November 2019, the therapeutic evaluation suggested that the tumor burden was significantly shrinking through eCT imaging, whereas left strabismus and instability of gait were detected on physical examination. Further, brain magnetic resonance imaging (MRI) revealed multiple metastasis in the right parietal lobe, left occipital lobe and cerebellum. This disease progression was followed by a protocol based on docetaxel $75 \mathrm{mg} / \mathrm{m}^{2}$ and carboplatin AUC $=5$ plus trastuzumab every 21 days in combination with planned simultaneous radiotherapy on December 18 ${ }^{\text {th }}, 2019$ (Table 1). Unfortunately, the symptoms, including bilateral visual loss and instability of gait, were increasingly deteriorating during this time, and they chose best supportive care (BSC) instead of any medical therapeutics and examinations in January 2020. She was alive in a poor performance status and the follow-up was terminated on August $1^{\text {st }}, 2020$.

Table 1: Clinical characteristics and disease course of the reported patient.

\begin{tabular}{|c|c|}
\hline Gender & Female \\
\hline Race & Asian Chinese \\
\hline Age at diagnosis & 37 years old \\
\hline Type of cancer & Invasive ductal breast cancer \\
\hline Clinical stage & cT4N3M1 Stage IV \\
\hline \multicolumn{2}{|r|}{ IHC } \\
\hline Primary disease & ER $(-)$, PR $(+, 90 \%)$, HER2 $(-)$, Ki67 $(+, 10 \%)$ \\
\hline Secondary disease & ER $(-), \operatorname{PR}(+, 90 \%), \operatorname{HER} 2(2+)$ \\
\hline \multicolumn{2}{|r|}{ FISH } \\
\hline the ratio of HER2 to CEP17 & 6.6 \\
\hline the ratio of average HER2 copies & 1.9 \\
\hline Metastatic sites & Multiple viscera, bone and soft tissue \\
\hline Molecular feature & TP53 mutation c.1010G>A (p. Arg337His) \\
\hline First-line therapy & Epirubicin $90 \mathrm{mg} / \mathrm{m}^{2}$, paclitaxel liposomes $175 \mathrm{mg} / \mathrm{m}^{2}$, intravenously, every 21 days, 4 cycles \\
\hline Therapeutic evaluation & Progression disease, newly diagnosed brain metastasis \\
\hline \multirow[t]{2}{*}{ Second-line therapy } & Docetaxel $75 \mathrm{mg} / \mathrm{m}^{2}$, carboplatin AUC $=5$, trastuzumab, \\
\hline & intravenously, every 21 days, 2 cycles \\
\hline Therapeutic evaluation & Recognized as progression disease, deteriorating symptoms \\
\hline
\end{tabular}

\section{Discussion}

This is a 37-year-old woman who was newly diagnosed as de novo stage IV breast cancer with synchronous metastases to stomach and bilateral ovaries. No apparent Initial symptoms were exposed other than anorexia and weight loss. Endoscopic biopsy confirmed the appearance of linitis plastic in addition to the integrity of mucous, whilst HER2 status conversed to amplification in the secondary lesion. Genomic profiles test showed TP53 germline mutation c.1010G>A (p. Arg337His). The overall clinical response to chemotherapy plus anti-HER2 targeted therapy, from this patient, was poor for rapid brain metastasis with the loss of vision and locomotion and the descending performance status.

Gastric metastasis from breast carcinoma is not a common phenomenon in clinical practice. On the basis of literature review [4-25], an incidence ranging from $1.7 \%$ to $6 \%$ was reported in the involvement of gastrointestinal metastases from primary breast cancer [26]. The average age of patients was 53 years (ranging, 38 to 84). Invasive lobular breast cancer occupied a higher risk, than 
that of ductal subtype, to metastasize in stomach with incidence of $69.1 \%$. The luminal subtype was supposed to hold the foremost rate of $92.1 \%$ and triple-negative (TN) also had a risk of $7.9 \%$, while gastric metastasis was not documented to occur in HER2 enriched subtype (Table 2).

Table 2: Clinical Characteristics of breast cancer and gastric metastasis from literature review.

\begin{tabular}{|c|c|}
\hline Characteristics & No. of Patients $(\mathrm{N}=46)$ \\
\hline Age at primary diagnosis (average, yr) & 53(ranging, 38 to 84 ) \\
\hline \multicolumn{2}{|c|}{ Breast cancer } \\
\hline \multicolumn{2}{|c|}{ Pathology } \\
\hline IDC & $13(30.9)$ \\
\hline ILC & $29(69.1)$ \\
\hline \multicolumn{2}{|l|}{ Subtype } \\
\hline Luminal & $35(92.1)$ \\
\hline HER2 enriched & 0 \\
\hline Triple-negative & $3(7.9)$ \\
\hline \multicolumn{2}{|c|}{ Gastric metastasis } \\
\hline \multicolumn{2}{|c|}{ Abdominal symptoms } \\
\hline Weight loss & $7(15.2)$ \\
\hline Abdominal pain & $14(30.4)$ \\
\hline Dysphagia & $6(13.0)$ \\
\hline Nausea & $6(13.0)$ \\
\hline Vomit & $5(10.9)$ \\
\hline GI bleeding & $8(17.4)$ \\
\hline Anemia & $6(13.0)$ \\
\hline Asymptomatic & $17(36.9)$ \\
\hline Others & $7(15.2)$ \\
\hline \multicolumn{2}{|c|}{ Subtype } \\
\hline Luminal & $27(90.0)$ \\
\hline HER2 enriched & 0 \\
\hline Triple-negative & $3(10.0)$ \\
\hline \multicolumn{2}{|c|}{ Subtype status } \\
\hline Consistency & $16(72.3)$ \\
\hline Inconsistency & $6(27.7)$ \\
\hline \multicolumn{2}{|c|}{ Comitant metastasis } \\
\hline None & $23(50.0)$ \\
\hline \multicolumn{2}{|c|}{ Yes } \\
\hline \multicolumn{2}{|c|}{ Visceral } \\
\hline Gastrointestine & $5(10.9)$ \\
\hline Ovaries & $2(4.3)$ \\
\hline Lung & $5(10.9)$ \\
\hline Liver & $6(13.0)$ \\
\hline Brain & $2(4.3)$ \\
\hline Bone & $11(23.9)$ \\
\hline Soft tissue & $8(17.4)$ \\
\hline Others & $3(6.5)$ \\
\hline
\end{tabular}


The initial symptoms of gastric metastasis tend to be unspecific. Furthermore, except for $30.4 \%$ proportions appeared abdominal pain, the initial symptoms tend to be far from recognition with the incidence of $36.9 \%$ obtained for asymptotic patients. The average time from primary disease to gastric metastasis was 6.6 years, of which $39.5 \%$ cases occurred within 5 years after the diagnosis of breast cancer and $18.6 \%$ proportions presented at diagnosis of de novo stage IV breast cancer. Of note, a long term was suggested that around one-third patients confirmed metastasis to stomach above ten years after the completion of adjuvant therapy for breast cancer (Figure 4). The distance interval beyond maybe 10 years, in addition to the imperceptible emergence, could further lead to the difficulty of diagnosis and therapy delay.

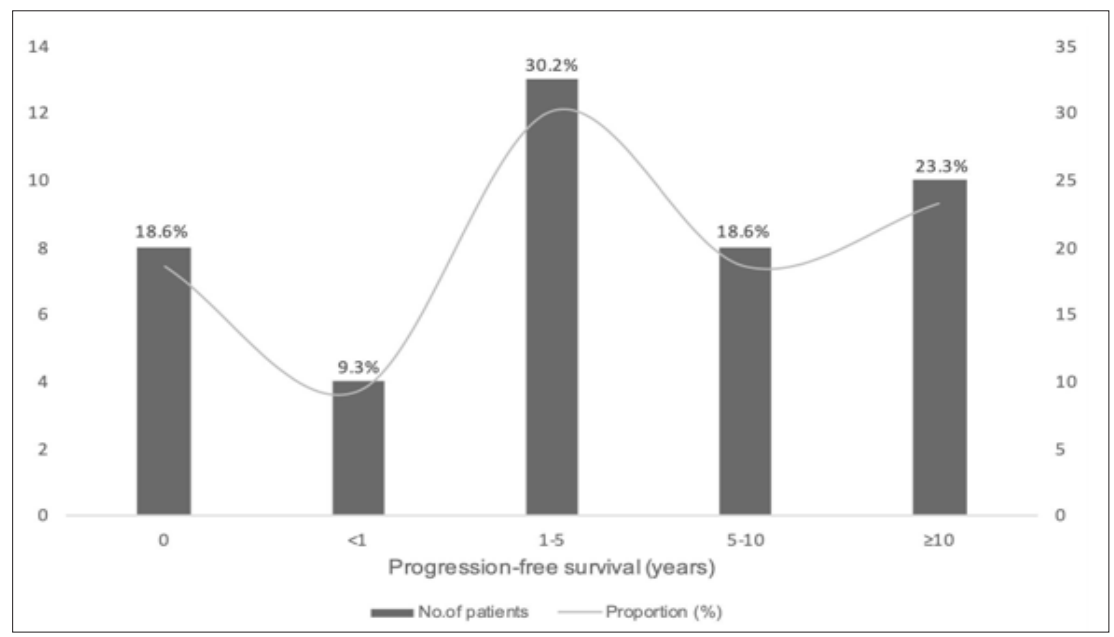

Figure 4: Time from primary breast cancer to gastric metastasis.

The diagnosis of gastric metastasis is dependent on endoscopic biopsy and histopathological examination. The most common endoscopic view is linitis plastic with the rate of $78.7 \%$, whereas the evident ulcer or polypus lesions occupy 19.3\%. However, linitis plastic is also a common endoscopic sign of primary gastric carcinoma at the advanced stage, which further increase the difficulty in antidiastole. Under this circumstance, more significance should be attached to the solid evidence acquired from immunochemistry analysis. GATA binding protein gene 3 (GATA3), gross cystic disease fluid protein-15 (GCDFP-15) and mammaglobins are the main diagnostic markers for primary breast cancer with the sensitivity of $32 \%-100 \%, 5 \%-74 \%, 7 \%-84 \%$, and the specificity of $71 \%$ 93\%, 85\%-100\%, 9\%-100\%, respectively [3,27]. Cytokeratin 7 (CK7) is estimated to entirely express positive in breast epithelial cells, which are negative, by contrast, in the primary carcinoma of the stomach. Conversely, CK20 is a fairly specific marker for gastrointestinal epithelial cells, yet with absence of expression in breast cancer. Combining the aforementioned markers could favor the clarification of the gastric origin.
A

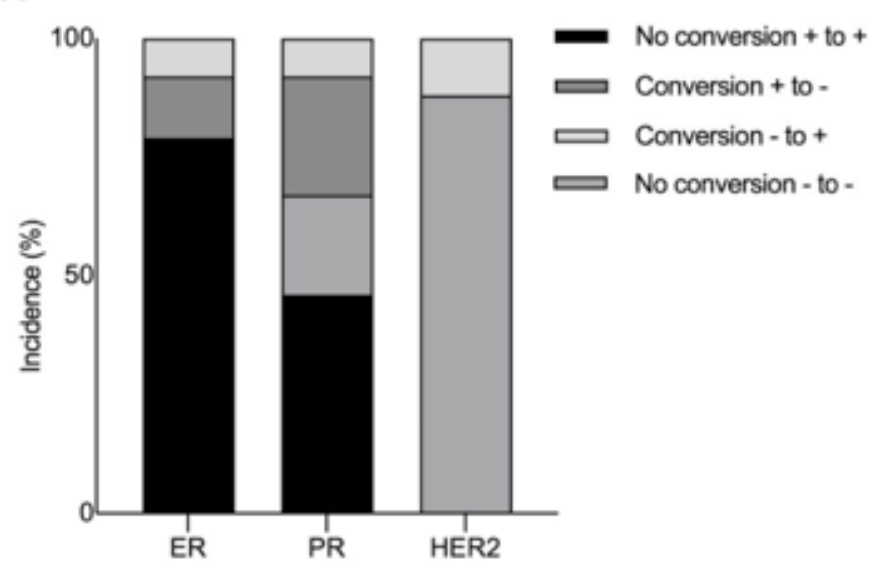

B

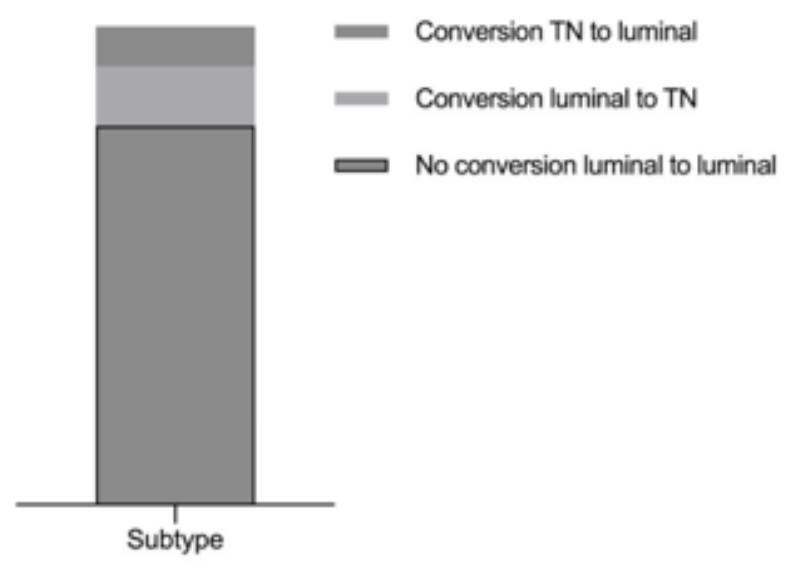

Figure 5: The conversion of ER, PR, HER2, and subtype in gastric lesions. 
The decisive role of hormonal receptors in the treatment for breast cancer could not be applicable to antidiastole, since a total of $72.3 \%$ proportion of gastric carcinoma remained in accordance with primary breast cancer in terms of endocrine-dependent subtype. Notably, the conversion of molecular receptors occupied a substantial incidence during the metastasis, with overall rate of $21 \%, 33 \%$, and $12 \%$ obtained for ER, PR and HER2 (Figure 5). Although the primary breast cancer was entirely HER2-negative, a $12 \%$ proportion of gastric lesion with HER2 expression conversed to positive, which enable the application of anti-HER2 targeted therapy and promise more survival benefit. In this respect, the test of hormonal receptors and HER2 expression were more meaningful to sequential therapy for metastatic disease. Moreover, in this report, the patient was first presented with breast cancer and synchronous gastric as well as ovarian and peritoneum disease, which was mimicking primary gastric carcinoma biological behaviors of implantation metastasis. Re-biopsy of secondary disease should be attached with more importance for not only differential diagnosis but also therapeutic chances.

Data on the following treatment were relatively consistent, which the entirety of patients received systemic treatment after the diagnosis of gastric metastasis, and chemotherapy, in combination with endocrine agents, was administrated for the aim of anti-cancer efficacy. Previous study suggested that surgical performance, rather than palliative surgical resection, could not significantly improve survival of patients [28]. However, there were significant differences in the prognosis of patients, which the time from gastric metastasis to death was ranging from one months to more than 42 months. No apparent factors from disease characteristics could account for the heterogeneity of survival, while more reasons could lie in the molecular mechanisms. The hallmarks of cancer are reflected in several aspects, where TP53 mutation plays a crucial role in the dysfunction of proliferation, metabolism and apoptosis, and could attribute to the poor prognosis of patients [29-31]. In this patient, saliva genetic test showed a missense mutation of TP53 with c.1010G>A (p. Arg337His). Although no genetic analysis was undergone due to the pessimistic economic conditions, this result could offer a solid clue to the aggressiveness of disease behaviors, especially of such an explosive progression at diagnosis. The intricate of malignant behaviors and the behind driving factors remains to be elucidated in this precision era.

\section{Conclusion}

Gastric metastasis from breast cancer is not common and may mimic primary gastric carcinoma, especially at the first presentation of primary disease with synchronous extensive abdominal metastases. The antidiastole mainly relies on the histopathological tests through re-biopsy, and the initial symptoms and endoscopic views tend to be unspecific in clinical observation. The conversion of molecular features should be focused and used to optimize therapeutic protocol. With the in-depth exploration of the molecular mechanisms, increasing biomarkers should be confirmed and contribute to clinical practice in the future.

\section{References}

1. Waks AG, Winer EP (2019) Breast cancer treatment: A Review. Jama 321(3): 288-300.

2. Si Y, Yuan P, Hu N, Xue W, Jie Ju, et al. (2020) Primary tumor surgery for patients with de novo stage iv breast cancer can decrease local symptoms and improve quality of life. Ann Surg Oncol 27(4): 1025-1033.

3. Schwarz RE, Klimstra DS, Turnbull AD (1998) Metastatic breast cancer masquerading as gastrointestinal primary. Am J Gastroenterol 93(1): 111-114.

4. Villa Guzmán J, Espinosa J, Cervera R, Delgado M, Patón R, et al. (2017) Gastric and colon metastasis from breast cancer: Case report, review of the literature, and possible underlying mechanisms. Breast Cancer (Dove Med Press) 9: 1-7.

5. Pectasides D, Psyrri A, Pliarchopoulou K, Floros T, Papaxoinis G, et al. (2009) Gastric metastases originating from breast cancer: Report of 8 cases and review of the literature. Anticancer Res 29(11): 4759-4763.

6. Jones GE, Strauss DC, Forshaw MJ, Deere H, Mahedeva U, et al. (2007) Breast cancer metastasis to the stomach may mimic primary gastric cancer: Report of two cases and review of literature. World J Surg Oncol 5: 75 .

7. Baa AK, Naik RD, Vanidassane I, Saurabh A, Shamim A, et al. (2020) Unusual gastric metastasis in triple-negative (Estrogen Receptor/ Progesterone Receptor/HER2neu Negative) GATA-binding protein 3-positive breast cancer. Indian J Nucl Med 35(1): 82-83.

8. Asmar N, Rey JF, Sattonnet C, Barriere J (2018) Gastric metastasis mimicking linitis plastica 20 years after primary breast cancer. A case report. J Gastrointestin Liver Dis 27(4): 469-471.

9. Ulanja MB, Taha M, Al-Mashhadani A, Bryce D, Marwah A, et al. (2018) Gastrointestinal bleed from erosive gastritis and duodenitis: A sentinel event of invasive lobular carcinoma of the breast and a diagnostic dilemma. Cureus 10(6): e2757.

10. Zullo A, Balsamo G, Lorenzetti R (2016) Gastric metastases from gynaecologic tumors: Case reports and review of the literature. Ann Transl Med 4(24): 483.

11. Hong J, Kim Y, Cho J, Sung W, Song E et al. (2019) Clinical features and prognosis of breast cancer with gastric metastasis. Oncol Lett 17(2): 1833-1841.

12. Tohfe M, Shami P, Aftimos G, Saade M (2003) Gastrointestinal metastases from breast cancer: a case report. South Med J 96(6): 624-625.

13. Ambroggi M, Stroppa EM, Mordenti P, Claudia B, Adriano Z, et al. (2012) Metastatic breast cancer to the gastrointestinal tract: report of five cases and review of the literature. Int J Breast Cancer 2012: 439023.

14. Zhang B, Copur-Dahi N, Kalmaz D, Boland BS (2014) Gastrointestinal manifestations of breast cancer metastasis. Dig Dis Sci 59(9): 23442346.

15. Soobrah R, Tsang F, Grassi V, Hirji H, Mallappa S, et al. (2015) Synchronous orbital and gastrointestinal metastases from breast cancer: A case report and review of literature. Case Rep Oncol Med 2015: 6.

16. Khan I, Malik R, Khan A, Salman A, Mehr Z, et al. (2017) Breast cancer metastases to the gastrointestinal tract presenting with anemia and intra-abdominal bleed. Cureus 9(7): e1429.

17. Zhong C, Fang X, Zhu L, Li D, Tang J, et al. (2019) Report of two cases and a systematic review of breast cancer with gastrointestinal metastasis. Turk J Gastroenterol 30(11): 997-1000.

18. Sudo G, Nasuno H, Nakachi K, Nakase H (2019) Gastrointestinal: Secondary gastric linitis plastica: A peritoneal recurrence of breast cancer. J Gastroenterol Hepatol 34(12): 2057. 
19. Güler SA, Şimşek T, Pösteki G, Alican G, Saffet Ç, et al. (2019) A very rare reason for gastric perforation, caused by gastric metastasis of breast cancer: Case presentation. Eur J Breast Health 15(1): 59-62.

20. Koike K, Kitahara K, Higaki M, Urata M, Yamazaki F, et al. (2014) Clinicopathological features of gastric metastasis from breast cancer in three cases. Breast Cancer 21(5): 629-634.

21. Waseda Y, Hayashi T, Kaneko S (2015) Gastric metastasis from breast cancer visualized by magnifying endoscopy with narrow-band imaging. Dig Endosc 27(6): 713

22. Kim H, Son M, Choi J (2018) Gastric metastasis from invasive lobular breast cancer, mimicking primary gastric cancer: A case report. Medicine (Baltimore) 97(13): e0258.

23. Hamada Y, Tanaka K, Hattori A, Mizuno T (2020) Gastric metastasis resembling linitis plastica 17 years after lobular breast cancer. Dig Liver Dis 52(2): 232 .

24. Abid A, Moffa C, Monga D (2013) Breast cancer metastasis to the GI tract may mimic primary gastric cancer. J Clin Oncol 31(7): e106-107.

25. Nazareno J, Taves D, Preiksaitis H (2006) Metastatic breast cancer to the gastrointestinal tract: A case series and review of the literature. World J Gastroenterol 12(38): 6219-6224.

26. Taal B, Peterse H, Boot H (2000) Clinical presentation, endoscopic features, and treatment of gastric metastases from breast carcinoma. Cancer 89(11): 2214-2221.

27. Gown A, Fulton R, Kandalaft P (2016) Markers of metastatic carcinoma of breast origin. Histopathology 68(1): 86-95.

28. EC McLemore, Pockaj BA, Reynolds C, Richard J, Jose L, et al. (2005) Breast cancer: Presentation and intervention in women with gastrointestinal metastasis and carcinomatosis. Ann Surg Oncol 12(11): 886-894.

29. Hanahan D, Weinberg R (2000) The hallmarks of cancer. Cell 100(1): 57 70.

30. Menendez D, Inga A, Resnick M (2009) The expanding universe of p53 targets. Nature reviews Cancer 9(10): 724-737.

31. G Zhou, Z Liu, JN Myers (2016) TP53 mutations in head and neck squamous cell carcinoma and their impact on disease progression and treatment response. J Cell Biochem 117(12): 2682-2692. 